\title{
Medical assessment of fitness to dive. Part I
}

\author{
Jarosław Krzyżak ${ }^{1}$, Krzysztof Korzeniewski ${ }^{2,3}$ \\ ${ }^{1}$ Polish Society of Hyperbaric Medicine and Technique, Gdynia, Poland \\ ${ }^{2}$ Department of Epidemiology and Tropical Medicine, Military Institute of Medicine, Warsaw, Poland \\ ${ }^{3}$ Department of Occupational, Metabolic and Internal Diseases, Institute of Maritime and Tropical Medicine, \\ Medical University of Gdansk, Poland
}

\begin{abstract}
Good physical and mental health is a prerequisite for anyone planning to scuba dive. A fitness to dive certificate for those willing to enter a scuba diving course as well as for active divers, either amateur or occupational, can only be issued if there are no medical contraindications to dive. It is usually within the competence of a diving instructor, a manager of underwater work or a physician to assess a person's mental and physical health and grant them permission to stay under hyperbaric conditions. The legal requirements for issuing a fitness to dive certificate are different for recreational and occupational divers. The part I of this article discusses the issues concerning medical assessment of fitness to dive for amateurs.
\end{abstract}

(Int Marit Health 2021; 72, 1: 36-45)

Key words: diving, health assessment, medical contraindications

\section{INTRODUCTION}

In Poland, recreational scuba diving courses are normally organized by privately-owned operators or sporadically by a few state-owned sports clubs affiliated to the Section of Underwater Activities at the Polish Tourist and Sightseeing Society. The training program offered by the state-owned institutions follows the guidelines proposed by the French Confederation Mondiale des Activites Subaquatiques (CMAS) and the Polish National Defense League, whereas scuba diving courses organized by privately owned operators are usually based on the procedures formulated by international diving organizations such as Professional Association of Diving Instructors (PADI), Scuba Schools International (SSI) or International Diving Association (IDA). Scuba diving courses for commercial divers, on the other hand, are organized by a number of state owned companies (e.g. the Polish Vessel Rescue Company, the Polish Register of Shipping and other shipping companies, shipyards or port authorities), by some privately-owned companies as well as by specialist units of the Polish Armed Forces, the Polish National Police and the State Fire Service. Good physical and mental health is a prerequisite for anyone planning to become a diver.
A fitness to dive certificate for those willing to enter a scuba diving course as well as for active divers (either amateur or commercial) can only be issued if there are no medical contraindications to dive. However right before a dive, it is normally within the responsibility of a diving instructor, a manager of underwater work or a physician to assess a person's mental and physical health and grant them permission to go underwater. The legal requirements for issuing a fitness to dive certificate are different for amateur and occupational divers [1].

\section{MEDICAL ASSESSIMENT OF FITNESS TO DIVE IN AMATEUR DIVERS}

A vast majority of scuba divers are amateurs. Nowadays, recreational diving is popular with people of all ages, including children and the elderly. Regardless of their age, all candidates for scuba diving are obliged to obtain a medical certificate confirming their good physical health and fitness to dive. In the past, entities authorized to issue such medical certificates were health care providers affiliated to sports medicine clinics, the Institute of Maritime and Tropical Medicine in Gdynia as well as military physicians. New

Prof. Krzysztof Korzeniewski, MD, PhD, Military Institute of Medicine, Head of the Department of Epidemiology and Tropical Medicine, Szaserów St. 128, 04-141 Warsaw, Poland, e-mail: kkorzeniewski@wim.mil.pl; Medical University of Gdansk, Institute of Maritime and Tropical Medicine, Department of Occupational, Metabolic and Internal Diseases, Powstania Styczniowego St. 9B, 81-519 Gdynia, Poland, e-mail: kkorzeniewski@gumed.edu.pl

This article is available in open access under Creative Common Attribution-Non-Commercial-No Derivatives 4.0 International (CC BY-NC-ND 4.0) license, allowing to download articles and share them with others as long as they credit the authors and the publisher, but without permission to change them in any way or use them commercially. 
legal regulations regarding scuba diving requirements (the Regulation of the Minister of Sport of August 17, 2006 on safety rules for practicing scuba diving, Journal of Laws of the Republic of Poland of 2006, item 1103) substantially simplified the procedure for obtaining certificates confirming lack of contraindications to diving [2]. Currently, a person who wants to dive should obtain an appropriate medical certificate, and in accordance with the applicable regulations, such a certificate may be issued by any physician. However, not many physicians are experts in the field of diving medicine; qualifying a person for a scuba diving course by a random and often incompetent physician carries a high risk of diving related accidents. In theory, ever potential diver should undergo a fitness to dive assessment by a medical practitioner before they enter a course. However, in order to promote the sport and increase its accessibility, many clubs and operators no longer require a fitness to dive examination or a certificate. Thus, the complete responsibility for any potential problems or accidents which may occur underwater lies with a diver alone. A physical examination byamedical doctorhas been replaced byaself-completed medical questionnaire, which is then analyzed by a diving instructor [3]. On the basis of the information provided by a candidate an instructor will decide whether or not a person can be declared fit to scuba dive [2]. This obviously places great responsibility in the hands of a scuba diving instructor. The instructor should not only be an expert in diving medicine who is fully aware of every single contraindication to scuba diving (like incidents of seizures, loss of consciousness, pulmonary edema) as well as any aspects that would temporarily disqualify a person from diving (e.g. middle ear infections, sinusitis, asthma, bronchitis and respiratory tract infections), but he must also be a skilled psychologist who has the ability to accurately assess a candidate's aptitude for diving before they start training (watch them carefully and look them deep in their eyes). The instructor must also be able to assess the risk of a potential diving accident and be able to recognize all the risk factors which might possibly lead to a diving-related incident, such as: poor physical condition or poor tolerance to exercise of a candidate, insufficient diving training, the presence of underlying health conditions that may aggravate under hypobaric conditions, the potential for a panic attack, poor planning of a diving session and a lack of enough air to complete a dive according to a pre-scheduled plan. As to date, Poland has not introduced effective legal regulations on medical requirements for recreational and sports diving [4]. Anyone interested in recreational scuba diving must rely on individual self-assessment of their health condition. The lack of legislation encourages diving associations to deal with the problems of diving fitness health assessment within their own capacities. In Poland, some diving organizations have been organizing training sessions for medical practitioners in the field of underwater and hyperbaric medicine; such training is offered by the Polish Hyperbaric Medicine and Technology Society and the Department of Hyperbaric Medicine and Maritime Rescue - the National Center for Hyperbaric Medicine at the Medical University of Gdansk. After completing a series of training sessions, participants are certified as diving physicians or club physicians. So far, several hundred physicians have completed the training, which means that anybody willing to start their adventure with scuba diving or those already practicing the sport should be able to consult with a competent diving specialist no matter which part of Poland they live in. A medical certificate stating that a person is medically unfit to dive must never be ignored. Individuals who have been declared 'unfit to dive' may apply for re-examination to the Head of the Diving and Scuba Diving Certification Board at the University Department of Maritime and Tropical Medicine in Gdynia. As a rule, commercial scuba divers are required to undergo a medical examination every 12 months but professionals taking part in scuba diving sports events should have their fitness to dive attested every 3 months $[2,5]$. Since scuba diving equipment is easily available to anyone and because formal restrictions for prospective divers are now more relaxed than they used to be in the past, some people fail to get proper training before they go scuba diving and are often completely unaware of the risks associated with this form of activity. From the medical point of view, it seems obvious that each prospective diver should get some insight into certain aspects of diving medicine. This will help them self-assess their fitness to dive and will hopefully reduce the risk of diving-related injuries. Until recently, a vast majority of scuba divers were all young and physically fit individuals, mostly under the age of 30 years. This, howev$\mathrm{er}$, has changed in recent years. Nowadays, scuba diving is popular with people of all ages. The activity is becoming increasingly popular among teenagers and some scuba diving fans decide to go diving with their children, in some cases no older than 10 years. The requirements for young people willing to engage in scuba diving are the same as for any other age group - they must be in a good physical and mental health and should be free from any contraindications to dive. As was mentioned before, the sport does not only attract the younger generation. In fact, more and more divers are either middle aged or retired people. Some older divers are in good physical health, while others will have certain preexisting conditions. Since a person's general health and level of fitness tends to decrease with age and because ageing is naturally associated with a number of degenerative processes affecting all organs and systems, older divers are recommended to undergo a careful medical examination and extensive diagnostic tests before they start 
scuba training $[4,6]$. It is often the case that the initial medical examination may reveal conditions of which a prospective diver has been unaware but which may be a relative or an absolute contraindication to scuba diving. As an example, undiagnosed asymptomatic cardiovascular conditions can unmask or aggravate when a person engages in strenuous physical activity (e.g. during a dive) causing a decrease in blood supply to vital organs. As was mentioned before, the age structure of individuals who practice scuba diving has changed over the past 30 years. This has had a direct influence on scuba diving fatalities statistics. The number of accidents in the 20-29 age group has decreased by a half in comparison to the period of 1987-1989 while, in the 50-59 and 60-69 age groups the percentage of diving-related accidents increased more than 5-fold. Currently, a certain proportion of divers are individuals in their 70 s or even 80 s; divers in this age category obviously run a much higher risk of a diving related accident or an illness in comparison to younger people. The average age of diving fatalities increased from 39 years in 1989 to over 54 in 2017. According to medical statistics the reason for $25-32 \%$ of deaths among divers are cardiovascular conditions. In light of the above, more than ever before, a fitness to dive assessment is of fundamental importance in the process of qualifying a person for recreational diving. Diving fitness health assessment in older divers as well as in divers with preexisting illnesses is a complicated issue. Its purpose is not only to declare all seemingly healthy individuals as 'fit to dive' and all those with preexisting conditions as 'unfit to dive', but rather to assess whether a patient's underlying conditions are a real contraindication to dive and to what extent will they affect a diver's health and safety underwater. The aim of a diving fitness health examination is also to determine safe diving limits for each diver $[6,7]$. In the past, good physical fitness was considered to be an absolute prerequisite for scuba diving, more recently however, scuba diving has become popular with all groups of people, even those with physical disabilities. Currently, poor physical fitness alone should not be considered an absolute contraindication to recreational diving, provided that a diver whose level of fitness is lower than normal acts in a reasonable manner and avoids unnecessary risk while staying underwater. Therefore, even people with physical disabilities or those with underlying conditions should be permitted to engage in recreational diving unless it may aggravate their underlying conditions. For many of them scuba diving might be an excellent form of active physical therapy [1].

\section{RESPIRATORY DISEASES}

Fitness to dive examination should primarily focus on the condition of the respiratory system since most diving related incidents and traumas are associated with pulmonary ede- ma caused by a respiratory arrest or abnormal retention of breathing gas in a lung or its part during ascent, even from the depth as low as 1-2 $\mathrm{m}$. This type of traumas may occur at any time, even during the very first dive session when a person is breathing compressed air. Any condition which is likely to increase the risk of air-trapping (i.e., abnormal retention of gas in lungs) during ascent or affect the lung volume during a dive should be considered an absolute contraindication to scuba diving. It is essential that each prospective diver should have a chest X-ray performed prior to their first dive. Amateur diving is contraindicated in patients with airway obstruction or emphysema which is visible on a chest X-ray, patients who are susceptible to spontaneous pneumothorax, patients who have recently undergone a thoracic surgery, as well as those with asthma, bronchitis or pneumonia. Because these conditions change the respiratory mechanics, their presence increases the risk of a pulmonary barotrauma during a dive in response to rapid changes in the intrapulmonary pressure $[4,8,9]$. Medical conditions which are characterized by increased mucus secretion, such as asthma, bronchitis, pneumonia, and bronchiectasis also increase the risk of air trapping. Excessive mucus secretion is also seen in heavy tobacco smokers [1].

Asthma is characterized by inflammation of the bronchial mucosa, paroxysmal bronchospasm and over-secretion of mucus in the respiratory tract. Inflammatory lesions cause the so-called bronchial hyper-responsiveness, which increases the risk of a bronchospasm. An asthma attack may be triggered by exposure to allergens or cold air, physical exercise, stress, over-excitement, infections or exposure to air irritants - many of these factors cannot be avoided while scuba diving. Asymptomatic asthma may be associated with persistent subclinical bronchospasm which increases the potential for a sudden asthma attack in case of exposure to any of the above mentioned irritants. Some divers might have had childhood asthma which resolved later in life, others will have allergic rhinitis or seasonal hay fever. All of them run a higher risk of an asthma attack while scuba diving.

Physical exercise alone may be the cause of about $90 \%$ of asthma attack cases but breathing dry and cold air from cylinders is also likely to trigger an asthma attack. In order to resolve any doubts, before taking the final decision concerning fitness to dive of a person suspected of bronchial hyper-reactivity, it is recommended to consult a pulmonologist and perform an exercise or a cold air challenge test. Individuals with chronic asthma may be declared fit to dive provided that they are well controlled with steroid drugs and have normal results on the spirometry test.

Owing to the fact that each year there is a growing number of asthmatic divers, British researchers under the guidance of prof. Elliott attempted to study this complex 
issue and find a solution to the problem. They have concluded that certain asthmatics can be allowed to scuba dive without running the risk of being exposed to diving-related illnesses or injuries. They have found that the prevalence rate of pulmonary barotraumas in asymptomatic asthmatics was not higher than in non-asthmatics. This led them to a conclusion that asthmatic patients with normal lung function parameters, at rest and post-exercise, may scuba dive safely provided that their forced expiratory volume (FEV) in 1 second on a spirometry test is more than $75 \%$ of the predicted $\mathrm{FEV}_{1}$ value $-\mathrm{FEV}_{1}>75 \%$. Elliott [8] also believe that patients with chronic asthma who are well-controlled with steroid drugs may be medically cleared to dive, provided that they have normal spirometry results. In contrast, active asthma constitutes an absolute contraindication to scuba diving. An asthma attack associated with a bronchospasm and overproduction of mucus in the airways prevent normal ventilation and increase the potential for air trapping which in turn increases the risk of a pulmonary barotrauma and cerebral gas embolism.

Bronchitis and pneumonia are common respiratory illnesses that are characterized by increased mucus secretion and the presence of inflammatory exudates which increase the risk of air trapping. An active infection definitely disqualifies a person from scuba diving. Occasionally, severe pneumonia may result in permanent changes in the lung structure, such as pulmonary fibrosis, cavities, emphysema, and calcifications. The lesions may obturate the bronchi and impair the lung function and its flexibility. Parts of the affected lung are generally more susceptible to injuries and air trapping. After an episode of pneumonia or bronchitis, a person should not resume scuba diving for at least a month after all the symptoms have disappeared. Before they return to diving they should consult a physician and have a chest $\mathrm{X}$-ray performed. Taking a break from scuba diving will be necessary to recuperate and clear the respiratory tract of the remains of mucus whose presence may increase the risk of small airways obstruction. Susceptibility to spontaneous pneumothorax is an absolute contraindication to scuba diving. It is a rare disease (with prevalence rate of about 5 cases per 100,000 people) that is most often seen in younger, healthy men; women are generally at a lower risk of spontaneous pneumothorax. The disease is more common in tobacco smokers. The direct cause of spontaneous pneumothorax is a rupture of pulmonary blebs and a tear in the visceral pleura and the subjacent lung structure. Blebs are usually small and not visible on chest X-ray. Recurrent episodes of spontaneous pneumothorax affect about $50 \%$ of individuals. It is well understood that if a prospective diver is susceptible to spontaneous pneumothorax under normal atmospheric conditions they will be even more vulnerable to such inci- dents under hypobaric conditions. Individuals with a history of thoracic surgeries, trauma-induced chest hematoma or pneumothorax should be disqualified from scuba diving. The formation of post-operative or post-traumatic pleural adhesions changes the respiratory mechanics and may impair normal breathing. Although the symptoms may not be clearly visible, changes in the lung volume during a dive, especially when holding one's breath, can cause further damage in the affected lung $[1,4,8,9]$.

\section{CARDIOVASCULAR DISEASES}

A routine fitness to dive assessment may reveal certain cardiovascular conditions. A physical examination, for example, can detect cardiac murmur or arrhythmias, while the electrocardiogram reading can reveal other abnormalities. If a prospective diver is diagnosed with any cardiovascular conditions, they will require further tests. In such cases, it is recommended to perform an echocardiogram, Holter electrocardiogram (a 24-hour test) and possibly a cardiac stress test. Once all the tests have been performed, a prospective diver should be referred to a cardiologist. In general, scuba diving is contraindicated in patients with a cardiovascular condition, patients with poor exercise tolerance, a positive cardiac stress test or any other abnormalities revealed by the standard cardiac tests.

Congenital heart defects that allow the blood to flow between the right and left chambers of the heart are an absolute contraindication to scuba diving. A person with the above mentioned conditions may only be allowed to dive provided that these defects have been corrected surgically in childhood, they report no cardiovascular symptoms and the results of cardiological and pulmonary tests show no abnormalities. Patients with asymptomatic valvular incompetence may be allowed to scuba dive, provided that they have normal results of electrocardiography and echocardiography. In older patients, it will be necessary to check whether valvular incompetence has not resulted from a more serious heart condition. If such a condition is confirmed by the echocardiogram the individual cannot be declared fit to dive. Any form of valvular stenosis disqualifies an individual from scuba diving. Individuals with asymptomatic mitral valve prolapse (a condition which affects approximately $5-10 \%$ of the global population) may be declared fit to dive unless they have reported symptoms such as palpitations, arrhythmias, chest pain or syncope, in such cases scuba diving will not be possible. It is important to remember that the presence of patent foramen ovale (PFO, a failure of the foramen ovale in the atrial septum to close) may significantly increase the risk of diving-related illnesses and affect diving safety. Although the condition is quite common and affects between $20 \%$ and $34 \%$ of the global population, prospective divers are not routinely screened for PFO and its presence 
is not considered a contraindication to scuba diving. Diving with PFO carries a certain level of risk - if the right atrium pressure exceeds the left atrium pressure during a dive, especially if a diver has not followed the recommended decompression procedures, gas bubbles are released into the arterial circulation causing arterial gas embolisms.

Abnormal electrocardiogram does not always mean that a patient has developed a cardiovascular disease, in some individuals it can signal sinus bradycardia or right bundle branch block.

Bradycardia, i.e., heart rate below 60 beats per minute is a condition associated with exercise-induced adaptation of the heart muscle. In athletes, bradycardia is normal and should not be considered a contraindication to scuba diving. In older patients, especially those who are not physically active, bradycardia is rather a signs of a heart condition and/or the effect of using cardiovascular medications. Such individuals should be consulted by a cardiologist and the decision concerning their fitness to dive should be taken with caution.

Right bundle branch block and incomplete right bundle-branch block is commonly seen in young physically active individuals and is not normally considered a contraindication to diving. The conditions are not associated with any heart disease and cause no signs or symptoms. In some patients, the ECG may reveal a brief resolution of the block when performing the Valsalva maneuver or during exercise. Patients with complete or incomplete right bundle branch block can safely engage in diving activities, but before they are medically cleared to scuba dive they need to be referred for an exercise tolerance test. Left bundle branch block is common in older people. The presence of left bundle branch block is indicative of a cardiovascular condition and is associated with a higher risk of a cardiac event; therefore it is considered an absolute contraindication to scuba diving. Wolff-Parkinson-White (WPW) syndrome is a condition associated with episodes of paroxysmal tachycardia, i.e., supraventricular tachycardia, atrial fibrillation or atrial flutter. WPW syndrome is an absolute contraindication to diving; a tachycardia attack under water may result in a loss of consciousness which, in consequence, may lead to drowning. For the same reason, candidates for divers with a history of paroxysmal supraventricular tachycardia (regardless of its cause) cannot be declared fit to dive. Individuals under 35 years of age may be conditionally allowed to scuba dive providing they have never fainted during a tachycardia attack, they have not been taking any cardiac medications and have not had an attack in the last six months. First-degree atrioventricular (AV) block is quite common in professional sports people as well as in some healthy individuals. A candidate diver with first degree $\mathrm{AV}$ block should first consult with a cardiologist; such pa- tients can be declared fit to dive, provided that the results of the cardiac stress test and echocardiography show no abnormalities. Second-degree and third-degree AV block is always a sign of a cardiac condition and therefore it is an absolute contraindication to diving.

Ischemic heart disease (IHD) is the leading cause of premature mortality for both men and women and of excess mortality for middle-aged men. The most dangerous consequence of IHD is myocardial infarction (MI). A history of a MI, angina or cardiac arrhythmias which have resulted from IHD are an absolute contraindication to diving. IHD is often associated with dangerous arrhythmias, which in $20 \%$ cases are responsible for the so-called 'sudden cardiac death'. IHD was found to be the largest single cause of sudden cardiac death in scuba divers. When assessing fitness to dive of individuals in their 40s or older, special attention should be given to IHD risk factors (high total cholesterol level, past history of IHD, family history of IHD, MI or sudden cardiac death in parents or siblings, arterial hypertension, diabetes, significant obesity). Prospective divers over the age of 40 with IHD risk factors or a history of a cardiac condition should have a cardiac stress test performed before they are declared fit to dive. If the test result is negative and a diver can reach a work rate of 13 metabolic equivalent level, which is approximately $45 \mathrm{~mL} \mathrm{O}_{2} / \mathrm{kg} / \mathrm{min}$, they can be considered fit for recreational diving. In doubtful cases, further non-invasive cardiac evaluation will be necessary, including echocardiogram and exercise scintigraphy. The presence of cardiac symptoms, exercise intolerance, a positive result of the cardiac stress test or any abnormalities revealed by cardiac tests disqualify a person from scuba diving. Other cardiac contraindications for scuba diving include: pacemaker implantation, artificial heart valves insertion, a history of coronary angioplasty or coronary artery bypass grafting. A diver who has had a MI should not return to scuba diving for 12 months after the cardiac event. After this period, a patient will need to undergo a thorough cardiovascular evaluation to assess his exercise tolerance and long-term complications of the infarction. In general, an episode of Ml decreases a diver's tolerance to exercise, stress and cold. A diver who effectively controls his blood pressure with medication and has normal exercise electrocardiography results can re-apply for a fitness to dive certificate. In some cases additional cardiac exams, such as echocardiogram or coronography will have to be performed. Complications associated with MI such as electrical conduction abnormalities may disqualify a person from scuba diving. Also pacemaker implantation is a contraindication to resuming any diving-related activities. Because they will require chronic anticoagulation treatment, patients with artificial heart valves should not be medically cleared to return to scuba diving. It must be 
remembered that the diving reflex may aggravate the existing arrhythmias and conduction abnormalities. The recent advances in medicine have enabled surgical treatment of coronary arteries. Although angioplasty and coronary artery bypass grafting significantly improve tolerance to exercise and general condition in patients with coronary artery disease or after $\mathrm{Ml}$, clearing such persons to return to scuba diving carries a certain level of risk due to the progressive atherosclerotic process.

Approximately $45 \%$ of people aged between 45 and 64 suffer from arterial hypertension. In candidates for diving it will be necessary to check whether or not arterial hypertension has caused permanent damage to the heart, kidneys and the fundus of the eye. The presence of left ventricular hypertrophy or left ventricular dilation disqualifies an individual from scuba diving. Individuals whose condition is effectively controlled with anti-hypertensive medications can be declared fit for recreational diving. However, if blood pressure cannot be normalized, a patient should not be medically cleared to scuba dive. Physical effort, stress and cold water exposure during a dive may potentially cause a significant increase in blood pressure. The risk of a stroke or a heart attack is much higher in those divers who are at an older age and have been diagnosed with arterial hypertension; therefore, such candidates should be disqualified from scuba diving.

Arterial and venous diseases are another major contraindication to scuba diving, because they are known to limit the physical capability of a diver. The exposure to cold water causes constriction of blood vessels and a reduction in blood supply to muscles. Arterial insufficiency in the lower limbs is a condition which will have a negative effect on a diver's swimming efficiency. Varicose veins are not considered to be a contraindication to scuba diving, provided that the condition is not associated with venous insufficiency or thromboembolism. Until only a few years ago, cardiovascular conditions were considered an absolute contraindication to dive. Currently, it is not uncommon for diving specialists to consult older divers with underlying heart conditions, which have developed as a result of many years of diving. An experienced physician should be able to determine safe diving depth limits for those who begin scuba diving at an older age. A large number of prospective divers are over 40 years old, sometimes even older than 60 . Divers in this age category will be more likely to have an underlying heart problem or hypertension in comparison to younger divers. Unless they have a serious cardiovascular disease, people over the age of 50 cannot be declared unfit to dive solely because of advanced age. Divers at an older age are likely to have lower physical fitness and exercise tolerance in comparison to younger divers. This, coupled with cardiovascular conditions may lead to a sudden aggravation of the underlying diseases and increase the risk of a sudden cardiac death in water, drowning or sustaining a diving-related injury. Elderly candidates with pre-existing cardiovascular conditions must be made aware of the risk of sudden cardiac death which may occur while scuba diving and of the potential risk for their dive buddy - in emergency situations a younger diver will have to risk his own life in order to save his older partner. When assessing their fitness to dive, a physician will not only have to consider the risk of a sudden aggravation of an underlying cardiovascular disease, but also the effects of immersion itself, stress, physical effort and cold temperature on the functions of the cardiovascular system. It cannot be assumed that every elderly diver will only engage in recreational diving, i.e., swim at a slow to moderate pace at shallow depths. During any dive, difficult conditions and circumstances can arise which may require vigorous exercise. In case of an emergency, a diver may be pushed to their limits in order to save himself or help his dive buddy, for example, he may have to swim against a strong current or a tide, he may become entangled in a fishing net, come across dangerous marine animals or have to deal with a failure of scuba diving gear. All these unforeseen circumstances rapidly increase the physical and mental strain, and may potentially turn out life-threatening for an elderly diver. Each diver should have sufficient cardiovascular reserves to be able to cope with unexpected emergencies without risking complete exhaustion or loss of consciousness. It is important to note that as they grow older people become more sensitive to temperature changes. Therefore, older divers will be more susceptible to dehydration when travelling to tropical regions or hypothermia when diving in seemingly warm waters. Both dehydration and hypothermia affect the function of the cardiovascular system and may trigger a cardiac event. Additionally, the stress associated with diving increases blood pressure and can cause tachycardia, which in patients with cardiovascular risk factors may result in acute MI leading to a sudden cardiac death. According to the statistics, as much as $25-32 \%$ of diving-related deaths in divers at an older age are associated with cardiac events [1, 4, 8-13].

\section{DISEASES OF THE EAR AND PARANASAL SINUSES}

Chronic otitis media or sinusitis, marked stenosis of the auditory canal, permanently perforated tympanic membrane or any recent surgeries of the ear constitute absolute contraindications to scuba diving. The presence of the above listed conditions will make it difficult or even impossible for a diver to equalize pressure in the ear and in the sinuses. Diving in a wet suit with perforated eardrum may lead to irritation of the labyrinth and disorientation under water. Eustachian tube dysfunction and edema within the nasopharynx are usually caused by frequent infections of the 
upper respiratory tract or by otitis media. The abnormalities increase the risk of the barotrauma of the ear or paranasal sinuses. Other common risk factors for barotrauma include: allergies, tobacco smoking, overuse of nasal sprays or nasal drops, polyps or other abnormalities within the nasal cavity. All these conditions may prevent normal ventilation of paranasal sinuses. People with childhood history of acute otitis media may have difficulties in normal ventilation of the ear at an older age which will increase their potential for an ear barotrauma. Congenital defects, such as cleft palate or bifid uvula are often associated with anomalies of the Eustachian tube. Surgical correction of the defects does not necessarily restore the function of the Eustachian tube. Patients with the above defects should be tested for the patency of the Eustachian tubes before they are medically cleared to scuba dive. If a diver had suffered perforation of the tympanic membrane they can only be allowed to resume diving after it has reattached and the ear function has returned to normal. Diving in a dry suit protects the middle ear from flooding and because it prevents the labyrinth from exposure to thermal irritation it allows a diver to stay underwater even if the eardrum ruptures. A history of the mastoid process or the auditory ossicles surgery disqualifies a candidate from diving due to a high risk of damage to the inner ear. People who often develop inner ear infections or Meniere's disease which manifest with dizziness should not be allowed to undertake scuba diving. Dizziness is often accompanied by nausea and vomiting and may potentially lead to drowning. Some divers may experience dizziness during the ascent because of asymmetric pressure equalization in the middle ear. If dizziness occurs on each ascent, or if it is severe, recreational diving should be discontinued. A sinus $\mathrm{X}$-ray and a consultation with an otolaryngologist might be useful when assessing fitness to dive in prospective divers with a history of illnesses of the ear, nose or sinuses. However, the final decision can be left to the divers themselves. If they have problems with equalizing pressure during their first diving session or develop a severe headache or an earache while diving in shallow waters they are very likely to discontinue scuba diving anyway. A fitness to dive assessment in divers who have suffered a middle ear or inner ear barotrauma should be performed by an otolaryngologist. Such patients may be allowed to return to scuba diving provided that the tympanic membrane has healed and vestibular disorders (irritation of the inner ear) have resolved. If a diver is to dive in a dry suit only, he may conditionally be allowed to return to scuba before the eardrum has healed $[1,4,6,14,15]$.

\section{EYE DISEASES}

Good visual acuity and normal field of vision are essential for maintaining safety and sense of direction underwater. Having good eyesight is essential to read the screen of a dive computer, a depth gauge, a watch, a compass or decompression tables. The visibility underwater is often limited because of water pollution or because there is not enough sunlight. Color blindness should not be considered a contraindication to scuba diving, as it has no direct influence on a diver's safety. There are some ocular conditions, however, which will disqualify a candidate from scuba diving. These include any severe inflammatory conditions of the eye or its protective apparatus manifesting with pain, disturbed visual acuity, diplopia or photophobia. If such conditions occur, a prospective diver cannot be declared fit to dive until the disease is cured and all symptoms subside; such individuals will need to consult an ophthalmologist before they can be declared medically fit to dive. Visual acuity disorders are increasingly common nowadays. A growing number of young scuba diving candidates have some vision defects which have to be corrected by wearing prescription glasses or contact lenses. Vision defects are found in nearly all candidates over the age of 50 . When consulting individuals with decreased visual acuity, it will be necessary to determine whether scuba diving will be safe in their case and decide which forms of scuba diving activities can be recommended to an individual person. There are several ways to improve visual acuity underwater. A diver might, for example, wear soft contact lenses or use a special scuba mask fitted with corrective lenses.

Since the eyeball and the fluid within are not compressible, the eye is not affected by pressure changes. An injury to the eye or rupture of the cornea may only occur with facial barotrauma, i.e., a mask squeeze which occurs if a diver fails to exhale all the inhaled air during a descent. For this reason, a history of eye surgery is not a contraindication to recreational scuba diving. In general, individuals who have had an eye surgery are not recommended to go scuba diving for 2-3 months after the surgery or until the cornea has healed. People who have undergone a surgery to the eyelids, conjunctiva or eye muscles should be discouraged from scuba diving for 2-3 weeks after the surgery. Divers at an older age are more likely to have serious vision problems or eye diseases, such as cataracts or glaucoma. In fact, glaucoma is quite a common condition in middle aged and elderly people. The above conditions are not normally considered to be an absolute contraindication to scuba diving, unless the loss of vision is so profound that it could threaten diving safety. Divers who have had a glaucoma or cataract surgery can be allowed to resume scuba diving 3 months after the operation. Divers who have had any visual disturbances following an episode of a decompression sickness or arterial gas embolism should be strongly discouraged from further diving $[1,4,6]$. 


\section{NEUROLOGICAL DISEASES}

A history of seizures with a syncope as well as a history of transient ischemic attack or a stroke is considered to be absolute contraindications to scuba diving. The presence of the above listed conditions creates a significant risk for the safety of a diver himself as well as people around him. A typical tonic-clonic seizure which is associated with respiratory arrest creates a high risk of drowning or pulmonary barotrauma. During a pre-dive assessment particular attention should be paid to: a past history of head trauma, concussion, post-traumatic intracranial hematoma, skull fractures, loss of consciousness or memory disorders lasting more than 24 hours, severe infections presenting with seizures. In patients with a history of febrile seizures, the risk of non-febrile seizure recurrence has been estimated at $13 \%$. Such patients will require a pre-diving assessment by a neurologist and an electroencephalography test. If the test reveals no abnormalities, a patient can be medically cleared to dive recreationally. The assessment of divers who have experienced a head trauma but would like to return to scuba diving is a separate issue. Whether or not a person may be cleared to resume diving will primarily depend on trauma severity and the presence of residual neurological symptoms. Post-traumatic seizures occur in approximately $90 \%$ of patients who suffered a head injury. In such cases scuba diving should be discontinued. Divers who have suffered a mild head injury which has caused a brief loss of consciousness and memory loss may return to scuba diving after a minimum of 6 weeks after the injury provided that the neurological assessment reveals no deficits, their electroencephalography is normal and they do not require anticonvulsants. If head trauma results in a loss of consciousness lasting a longer period and a memory loss of more than three weeks, a person should not resume diving for a minimum of 12 months after the injury.

Another group of patients who should not be medically cleared to scuba dive are those who are more susceptible to syncopal episodes triggered by exposure to stress. Such individuals are likely to experience weakness, dizziness, excessive sweating, hot flushes and hypoglycemia when confronted with a stressful situation. Because it is essential that a diver remains conscious underwater at all times, re-current episodes of stress-related syncope are considered an absolute contraindication to scuba diving. Patients with a history of a stroke, even if it was mild and with minor residual symptoms only, must be disqualified from recreational scuba diving. If a stroke has been the effect of a vascular disease, there will be an increased risk of a vascular, cerebral or cardiac event during a dive. In very rare cases, a patient with a past history of a stroke episode may be cleared to take up less demanding scuba diving activities (only recreationally) provided that the risk of a recurrent stroke has been ruled out by specialist tests and that they have adapted to their functional limitations.

Chronic radiculopathy and degenerative disc disease are conditions commonly seen in the middle-aged and the elderly people. They usually present with chronic pain at multiple sites. If the conditions are not associated with any physical disability, they should not be considered a contraindication to diving. However, it must be remembered that swimming in cold water as well as lifting and carrying heavy objects, such as scuba gear, may cause recurrence of pain. In such a case, a person should discontinue diving until the symptoms have subsided. Patients who required surgical treatment of the above conditions should not resume scuba diving for a minimum of 2 to 3 months after the operation $[1,4,6,16,17]$.

\section{MENTAL DISORDERS}

Mental disorders which are regarded as absolute contraindications to scuba diving include: a history of psychotic episodes, anxiety or personality disorders, intellectual disability, alcohol addiction and substance abuse. Because there is no legal requirement for prospective divers to undergo a comprehensive fitness to dive assessment before they go scuba diving, some candidates may turn out to have an underlying mental disorder. In such cases, it will be up to a diving instructor to decide whether or not an individual is fit to dive. The instructor will be the only person who can judge the behavior and personality of a prospective diver and assess whether he is likely to make progress. Close supervision of candidates during their initial dives (their behavior, the way they handle equipment and react to the challenges involved in the training and unforeseen difficulties) will provide valuable information on their general diving aptitude. An experienced scuba diving instructor will usually be able to evaluate a person's diving fitness and aptitude during the very first training session. Candidates who are emotionally unstable should be discouraged from further training or any diving related activity. Those who exhibit anxiety or depression-related behaviors require close supervision during their preparatory training. If they are unable to control their fear and move to the next, more difficult stage of the training, they will be more susceptible to a panic attack while staying underwater. People with anxiety disorders often suffer from phobias, of which claustrophobia, i.e., the fear of confined spaces, is the most common. Other phobias which might be seen in prospective divers include: the fear of diving, the fear of water, the fear of depths, the fear of open spaces or of marine fauna and flora. When a diver experiences strong fear, he is more likely to lose self-control and panic. This can have grievous consequences as panic is the leading cause of death for scuba divers. On the other side of the spectrum, there is a group of natural risk-takers, people who are over- 
confident, self-centered and stubborn. Such candidates are likely to underestimate the danger associated with scuba diving and disregard the diving safety procedures. As a result, they will be more likely to experience a diving-related accident than other groups of divers. In addition, candidates who are too confident tend to ignore their diving buddy or the problems they may have during a dive, which can have tragic consequences. It should not be difficult for a diving instructor to identify such a person, as they are likely to brag about their past achievements, accidents or injuries. Unfortunately, it may be more difficult to dismiss such a candidate from a dive course. Once they have decided to complete the course, it will be difficult to dissuade them from doing so. In such situations, the only thing that the instructor can do is to supervise the 'unpredictable' candidate and ensure that he follows all the scuba diving safety regulations.

Most patients with psychosis (schizophrenia, bipolar disorder) experience periods of symptoms exacerbation and remission. During the period of remission they manifest no worrying symptoms and are likely to conceal their illness from other people. A detailed medical interview focusing on medications taken regularly, the family situation and work environment might be helpful in such cases. Patients on psychotropic medications cannot be medically cleared to scuba dive because the drugs impair a diver's performance underwater and exacerbate the symptoms of nitrogen narcosis. Quite a large number of recreational scuba divers report a regular use of medications; the most common include tranquilizers and anti-anxiety drugs. It must be emphasized that abuse of alcohol or of any antipsychotic medications is an absolute contraindication to scuba diving. Alcohol impairs judgment, delays reaction time and affects a diver's motor coordination. Diving under the influence puts a diver at a significant risk of having an accident or sustaining an injury; it also increases the risk of a barotrauma. Therefore, it is important that divers avoid alcohol not only immediately before a dive and in between the diving sessions but also the night before they go scuba diving (e.g. at team-building parties organized before a diving session). Similarly, drug addiction is considered an absolute contraindication to diving. The use of narcotic drugs endangers the safety of a diver and people around [1, 4, 6, 18, 19].

\section{DENTAL PROBLEMS}

A prospective diver needs to have all his front teeth to able to hold the scuba mouthpiece. Having a dental bridge or a dental prosthesis is not a contraindication to scuba diving, provided that they are firmly attached because a loose denture creates a risk of airway obstruction in case of an emergency. All prospective divers are recommended to visit a dental practitioner to have all their teeth fixed and filled before they start diving. Root canal treatment and loose fillings increase the risk of dental barotrauma. Multiple missing teeth and advanced caries lesions are considered a contraindication to scuba diving [1].

\section{GASTROINTESTINAL DISEASES}

Gastrointestinal system is the area of the body where gas can easily get 'air trapped'. Vomiting is a common symptom of some digestive tract disorders or illnesses; gastrointestinal conditions which are associated with an increased risk of vomiting and air trapping are an absolute contraindication to scuba diving. Vomiting underwater may potentially lead to drowning and the expanding gas trapped in the intestines may cause barotrauma.

Scuba diving, especially in a head-down position, promotes air swallowing, while the state of apparent "weightlessness" underwater has a negative effect on peristalsis, and may aggravate underlying gastrointestinal conditions. Gastroesophageal reflux disease is a significant contraindication to scuba diving because it puts a diver at risk of aspiration of the gastric contents. In mild cases, a patient may be conditionally cleared to scuba dive but he must be aware of the fact that scuba diving may cause aggravation of symptoms. Hiatal hernias which are asymptomatic do not disqualify a person from scuba diving. A major sliding hiatal hernia is considered an absolute contraindication to scuba diving as it increases the risk of distention and injury to the part of stomach displaced to the mediastinum (during ascent). Conditions associated with spasm of the cardia or pylori create a similar risk and therefore disqualify a candidate from diving. All factors which prevent gas being released from the stomach during ascent may cause gastric distention and rupture. For the same reason, obstruction of the small or large intestine is another contraindication to scuba diving. Patients with gastritis, gastric or duodenal ulcers or cholecystitis cannot be medically cleared to scuba dive until their condition has been successfully managed and all symptoms have resolved. Cholelithiasis is not a contraindication to dive. All hernias of the abdominal wall disqualify a candidate from diving until they are managed surgically. Lifting and carrying heavy objects, such as scuba gear increases the risk of hernia expansion and strangulation. Additionally, the presence of a hernia increases the risk of air trapping, with well-known consequences to the loop of the intestine filling the hernial sac. Patients who have had a hernia repair surgery can safely return to scuba diving after the post-operative wound has healed completely [1, 4, 6, 20].

\section{OTHER CONTRAINDICATIONS}

A number of active or prospective divers might have diabetes. The clinical course and the severity of the illness vary between individual patients. The main risk associated 
with diabetes is a sudden loss of consciousness due to hypoglycemia. Diabetic patients who manage their condition with diet and medication and have not reported any past episodes of hypoglycemia may be permitted to scuba dive. Patients who receive insulin therapy or those treated with oral medications but with a history of hypoglycemia must not be cleared to scuba dive.

Pregnancy and obesity are considered relative contraindications to recreational scuba diving. In general, pregnant women should be temporarily discouraged from any diving-related activity. Women who have had a natural birth are not recommended to go scuba diving for at least 4 weeks after they have given birth, while women who have had a Cesarean section should not scuba dive for 8-12 weeks after the delivery. Obesity does not only decrease general physical fitness but it may also increase the risk of a decompression sickness due to a high content of total nitrogen in the body (nitrogen is more soluble in fat than in other tissues). Candidates for scuba diving with any chronic conditions are recommended to have a fitness to dive examination before each dive. A candidate's fitness to dive will depend on the severity of their condition and how well it is controlled $[3,21]$.

In recent years, scuba diving has been increasing in popularity with people in their 50s. This is undoubtedly associated with a rapid growth in tourism globally, especially scuba diving tourism. Older people should be strongly recommended to undergo a careful medical examination and a fitness to dive assessment before they go diving. Candidates for recreational scuba diving must be made aware that the responsibility for their life and safety lies with themselves. It would be particularly dangerous if they tried to withhold any important information relating to their past or present medical history. It is the role of a physician, a specialist in diving medicine, to inform a patient of the conditions which are relative or absolute contraindications to scuba diving and of the potential health risk factors associated with this sport. Exposure to extreme environmental conditions (cold stress, underwater conditions, increased hydrostatic pressure, increased volume of respiratory gases) causes physiological and systemic changes which may unmask or aggravate certain pre-existing conditions [4, 9-13, 22, 23].

\section{REFERENCES}

1. Krzyżak J, Korzeniewski K. Medicine for divers [in Polish]. Publishing House 4Font, Poznań 2020: 25-37.

2. Regulation of the Minister of Sport and Tourism of August 17, 2006 on scuba diving safety regulations. Journal of Laws of the Republic of Poland of 2006, item 1103 [in Polish].

3. Undersea \& Hyperbaric Medical Society. Diver Medical Questionnaire. Available at: https://www.uhms.org/resources/recreational-diving-medical-screening-system.html. Accessed: 29 January 2021.

4. Bove AA. Medical evaluation for sport diving. In: Bove AA., Davis JC. Bove and Davis' Diving Medicine. Fourth Ed. Elsevier Inc., USA 2004: 519-532.
5. Regulation of the Minister of Health of September 17, 2007 on health requirements for occupational diving. Journal of Laws of the Republic of Poland of 2007, no. 199, item 1440 [in Polish].

6. Elliott DH. Medical evaluation for working divers. In: Bove A.A. Bove and Davis' Diving Medicine. Fourth Ed. Elsevier Inc., USA 2004: 533-545.

7. European Diving Technology Committee, Fitness to dive standards. Guidelines for medical assessment of working divers. www.edtc.org (Accessed: 24 June 2003).

8. Elliott DH. Are asthmatics fit to dive? Undersea and Hyperbaric Medical Society, Kensington MD. 1996.

9. Moon RE, Bove AA, Mitchell SJ. PFO statement. In: Denoble PJ, Holm JR. (eds.). Patent Foramen Ovale and Fitness to Dive Consensus Workshop Proceedings. Divers Alert Network, Durham, NC 2016: 156-160.

10. Lafay V, Trigano JA, Gardette B, et al. Effects of hyperbaric exposures on cardiac pacemakers. Br J Sports Med. 2008; 42(3): 212-216, doi: 10.1136/bjsm.2007.039552, indexed in Pubmed: 17768160.

11. Green RD, Leitch DR. Blood pressure and diving. J R Nav Med Serv. 1986; 72(1): 15-19, indexed in Pubmed: 3735185.

12. Vann RD, Lang MA. Recreational diving fatalities. Workshop proceedings. April 8-10, 2010. Durham NC, Divers Alert Network. 2011.

13. Childs CM. Loss of consciousness in divers - a survey and review. Proceedings. Medical Aspects of Diving Accidents Congress, Luxembourg 1978, pp. : 3-23.

14. Lechner M, Sutton L, Fishman JM, et al. Otorhinolaryngology and Diving-Part 1: Otorhinolaryngological Hazards Related to Compressed Gas Scuba Diving: A Review. JAMA Otolaryngol Head Neck Surg. 2018; 144(3): 252-258, doi: 10.1001/jamaoto.2017.2617, indexed in Pubmed: 29450472.

15. Lechner M, Sutton L, Fishman JM, et al. Otorhinolaryngology and Diving-Part 2: Otorhinolaryngological Fitness for Compressed Gas Scuba Diving: A Review. JAMA Otolaryngol Head Neck Surg. 2018; 144(3): 259-263, doi: 10.1001/jamaoto.2017.2616, indexed in Pubmed: 29450499.

16. Massey EW, Moon RE. Neurology and diving. Handb Clin Neurol. 2014; 120: 959-969, doi: 10.1016/B978-0-7020-4087-0.00063-2, indexed in Pubmed: 24365363.

17. Todnem K, Nyland H, Riise T, et al. Analysis of neurologic symptoms in deep diving: Implications for selections of divers. Undersea Biomed Res. 1990; 17: 95-107, indexed in Pubmed: 2321322.

18. Biersner RJ. Psychological standards for diving. In: Shilling CW, Carlston CB, Mathias RA. The Physician's Guide to Diving Medicine. Plenum Press, New York 1984: 520-529.

19. Morgan WP. Anxiety and panic in recreational scuba divers. Sports Med. 1995; 20(6): 398-421, doi: 10.2165/00007256199520060-00005, indexed in Pubmed: 8614760.

20. Vote D. Gastrointestinal issues - consider them before returning to diving. https://www.diversalertnetwork.org/medical/articles/ Gastrointestinal_Issues (Accessed: 29 January 2021).

21. Damnon F, de Rham M, Baud D. Should a pregnancy test be required before scuba diving? Br J Sports Med. 2016; 50(18): 1159-1160, doi: 10.1136/bjsports-2015-095770, indexed in Pubmed: 26944355.

22. Bennett PB, Moon RE. Diving Accident Management. Proc. 41st UHMS Workshop, Durham NC 15-16 Jan 1990. UHMS Publication No. 78; 1990: Bethesda.

23. DAN Annual Diving Report 2016 Edition. 2014 diving fatalities, injuries and incidents. Divers Alert Network, Durham, NC 2016. 